\title{
Distributed Simulation of Real Tunnel Junction Effects in Multi-Junction Solar Cells
}

\author{
P. Espinet ${ }^{\mathrm{a}}$, I. García ${ }^{\mathrm{a}}$, I. Rey-Stolle ${ }^{\mathrm{a}}$, C. Algora ${ }^{\mathrm{a}}$ and M. Baudrit ${ }^{\mathrm{a}}$ \\ ${ }^{a}$ Instituto de Energía Solar - Universidad Politécnica de Madrid ETSI de Telecomunicación, \\ Avda. Complutense 30, 28040 Madrid (SPAIN)
}

\begin{abstract}
In this paper, we present an improved 3D distributed model that considers real operation regimes in a tunnel junction. This advanced method is able to accurately simulate the high concentrations at which the current in the solar cell surpasses the peak current of the tunnel junction. Simulations of dual-junction solar cells were carried out with different light profiles and including chromatic aberration to show the capabilities of the model. Such simulations show that, under some circumstances, the solar cell short circuit current may be slightly higher than the tunnel junction peak current without showing the characteristic dip in the J-V curve. This behavior is caused by the lateral current spreading towards the dark regions, which occurs through the anode region of the tunnel junction.
\end{abstract}

Keywords: Photovoltaics, Concentrator, Tunnel Junction, Multi-Junction, Simulations, Solar Cells PACS: 88.40.fc, 88.40.jp, 81.05.Ea

\section{INTRODUCTION}

In the past decade, there have been marked advances both in efficiency of multi-junction solar cells (MJSCs) [1-4] and high-flux optical designs [5]. One of the key advances that has led to the manufacturing of these high efficiency multi-junction solar cells is the development of a low-loss interconnection system that minimizes the optical and electrical losses in the second or third subcells; namely a tunnel junction (TJ). However, when the current of the solar cell increases beyond the maximum tunnelling current of the tunnel diode (i.e., its peak current), a dip appears in the characteristic J-V curve, which reduces the conversion efficiency. Therefore, solar cell manufacturers have tried to ensure that the solar cell is within the tunnelling region when it operates around its nominal concentration level [6].

On the other hand, the photovoltaic concentrator design contains two problems: 1) the bundle-coupling problem for maximizing the acceptance-concentration product and 2) the prescribed irradiance problem for uniform irradiance in the active solar area. This is a difficult task and only partial solutions have been found. Many efforts have been carried out for making the irradiance on the solar cell more uniform [5] since solar concentrator optics often produce strongly inhomogeneous flux distributions[7]. Therefore, simulation models of MJSC are fundamental tools for predicting MJSCs behaviour under different flux levels and distributions. 3D simulations of MJSCs with a distributed model based on elemental circuit units are an excellent tool to represent the device under a wide range of concentration conditions [8]. However, to date and to the best of our knowledge, the tunnel junction has been modelled as a resistor [8] or even as a short circuit [9], which only takes the tunnelling region into account. This simplified model fails in situations that produce currents in the solar cell higher than the peak current of the tunnel junction. This paper presents a more precise model that operates without restrictions throughout the entire $\mathrm{J}-\mathrm{V}$ curve of the tunnel diode. Therefore, this advanced model is a very useful tool in the design of concentrator optics.

\section{DESCRIPTION OF THE 3D DISTRIBUTED CIRCUIT MODEL}

The philosophy of the 3D distributed model presented in this paper has been explained in detail elsewhere [10]. This approach divides the solar cell into elementary units and assigns each unit to a suitable circuit model, depending on its geometry and position in the solar cell area (shadowed, exposed, or perimeter). The complete solar cell can subsequently be modelled by an electrical circuit that is obtained by interconnecting each unit-circuit with its neighbours. The resulting equivalent circuit formed by thousands

CP1277, $6^{\text {th }}$ International Conference on Concentrating Photovoltaic Systems - (CPV-6)

edited by A. W. Bett, F. Dimroth, R. D. McConnell, and G. Sala

(C) 2010 American Institute of Physics 978-0-7354-0827-2/10/\$30.00 
of elements is resolved by using the SPICE circuit simulator software package. The circuits that describe the various solar cell regions are depicted in figure 1.

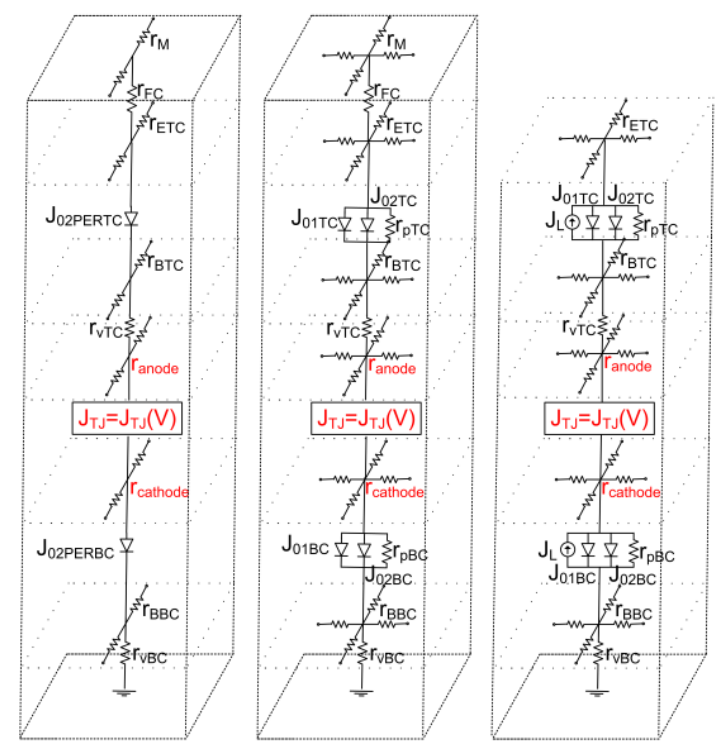

FIGURE 1. From left to right, the elementary unit cell of a perimeter area, dark area and illuminated area of a dualjunction solar cell are presented. In this advanced model, the tunnel junction is modeled with a component represented by an analytical expression and lateral resistances ( $r_{\text {anode }}$ and $r_{\text {cathode }}$ ). A detailed description of the components can be found elsewhere [10].

The key new aspects of the improved method are the following: a) the tunnel diode definition, which includes a full description of its $\mathrm{J}-\mathrm{V}$ characteristics; and $b$ ) the addition of lateral resistors surrounding the tunnel junction ( $r_{\text {anode }}$ and $r_{\text {cathode }}$ in figure 1). With these improvements we have developed a complete method for the modelling of multi-junction solar cells [12]. The J-V curve of the tunnel junction (see figure 2) can be introduced either as a table with values that are expected or measured, or as an analytical expression $\mathrm{J}_{\mathrm{TJ}}=\mathrm{J}_{\mathrm{TJ}}(\mathrm{V})$. In this paper, the complete static J-V characteristic of the tunnel diode junction was modelled using the analytical expressions presented in [11] which produce the $\mathrm{J}-\mathrm{V}$ curve depicted in 2 .

\section{DESCRIPTION OF THE SOLAR CELLS SIMULATED}

For the sake of clarity, a dual-junction GaInP/GaAs solar cell was used for the simulations. The simulated device includes the top and bottom cell features described previously [1], together with the tunnel junction sketched in figure 2. Briefly, the device is a high concentrator $\mathrm{GaInP} / \mathrm{GaAs}$ solar cell with an square active area of $1 \mathrm{~mm}^{2}$ and a front metal grid featuring an inverted square configuration that comprises eight evenly spaced fingers that are $3 \mu \mathrm{m}$ wide, i.e., a shadowing factor of $2.7 \%$, surrounded by a busbar that is $100 \mu \mathrm{m}$ wide. The AM1.5D low AOD short circuit current equals $13.5 \mathrm{~mA} / \mathrm{cm}^{2}$ for both subcells. We decided not to use the real tunnel junction described in [1] because its peak tunnelling current is so high that its effects become negligible for concentrations below 20.000 suns. Instead, we decided to use a tunnel junction more similar to that found in state-of-the-art MJSC, such as the one depicted in figure 2 with a $J_{p}=40.5 \mathrm{~A} / \mathrm{cm}^{2}$ which is equivalent to an irradiance of $3000 \mathrm{X}$ in the test device simulated.

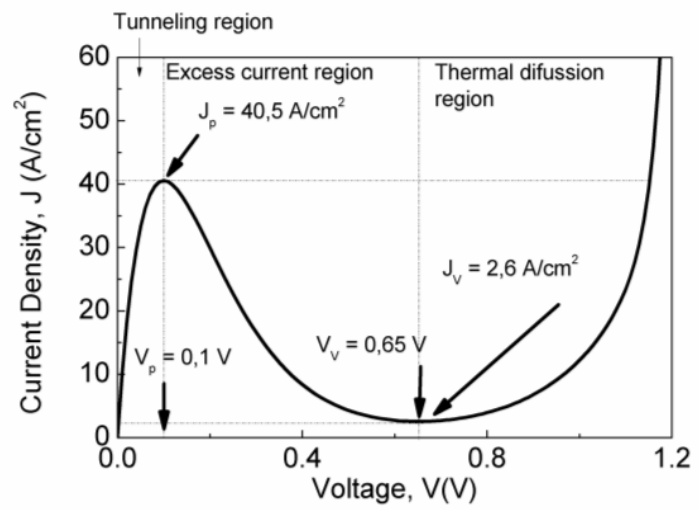

FIGURE 2. J-V curve of the tunnel junction used in the simulations presented in this paper. The characteristic parameters of the $\mathrm{J}-\mathrm{V}$ curve appear as labels in the figure: peak current density $\left(\mathrm{J}_{\mathrm{p}}\right)$, peak voltage $\left(\mathrm{V}_{\mathrm{p}}\right)$, valley current density $\left(\mathrm{J}_{\mathrm{V}}\right)$ and valley voltage $\left(\mathrm{V}_{\mathrm{V}}\right)$.

\section{SIMULATIONS UNDER UNIFORM IRRADIANCE}

A widely used method to determine the peak current of the tunnel diode in a multi-junction stack is to increase the irradiance on the cell and monitor the I$\mathrm{V}$ until a dip in the curve appears [6]. At this point it can be assumed that the short circuit current of the solar cell equals the tunnel diode peak current. According to the latter assumption, in our simulations, a dip in the I-V curve should be apparent at an irradiance level of just over $3000 \mathrm{X}$.

Figure 3 depicts the result of the simulations at two different concentration levels: $3090 \mathrm{X}$ and $3100 \mathrm{X}$. As expected, a dip in the I-V curve becomes apparent in the case of $3100 \mathrm{X}$. However, at $3090 \mathrm{X}$ no effects in the I-V curve were detectable. Therefore, this dualjunction solar cell can operate with illumination currents beyond the tunnel diode peak current without exhibiting the dramatic dip-related effects, up to at least $3090 \mathrm{X}$. False color maps at $0.5 \mathrm{~V}$ (before the 
dip) for the two irradiance levels, $3090 \mathrm{X}$ and $3100 \mathrm{X}$, have been depicted in figure 4 in order to understand the origin of this result. It has to be noted that experimental evidence in similar phenomena has been reported elsewhere [13].

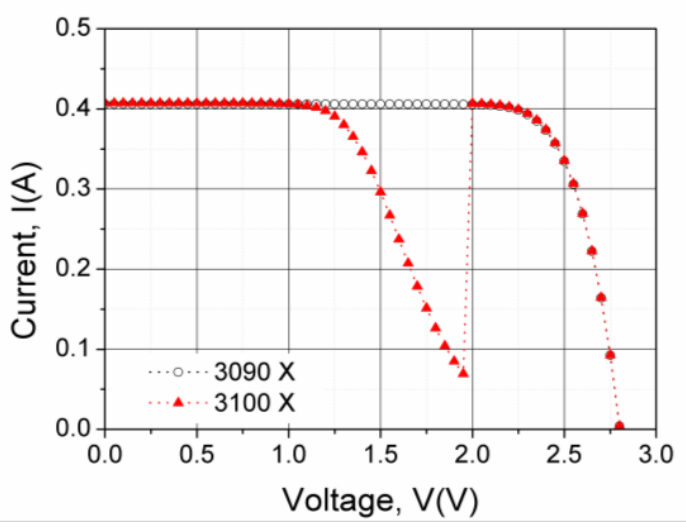

FIGURE 3. I-V curves for the dual-junction solar cell under uniform irradiance at different concentration levels.
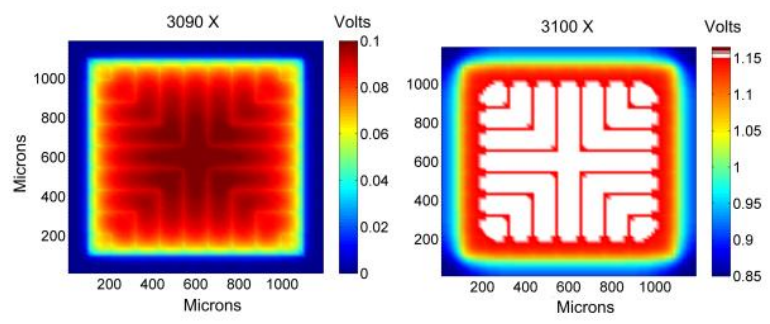

FIGURE 4. False color maps of the voltage drop in the tunnel junction when the solar cell is bias at $0.5 \mathrm{~V}$.

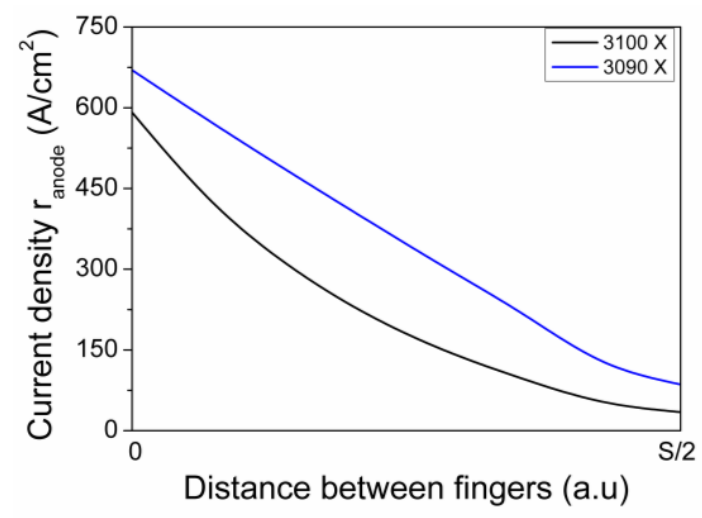

FIGURE 5. Lateral current through $r_{\text {anode }}$ versus half the distance between two adjacent fingers ( $\mathrm{S}$ is the finger pitch).

Figure 4 shows that voltage drop in the tunnel junction at $3090 \mathrm{X}$ is around $0.1 \mathrm{~V}$, therefore it is working in the tunneling region (figure 2) and the current through the junction is virtually equal to its peak current. This phenomenon is possible, despite the fact the photogenerated current is higher than the peak current because there is a lateral current density that diverts the excess current toward the dark areas where it can flow vertically (figure 5). At $3100 \mathrm{X}$ the voltage drop in the tunnel junction is around $1.15 \mathrm{~V}$ (plotted in white in figure 4), then the tunnel junction is working in the thermal diffusion region. At this voltage, the current through the tunnel junction exceeds the peak current in the illuminated area away from the fingers. The dip in the I-V curve is observed when the voltage drop the tunnel junction diminishes and consequently the current through the TJ decreases sharply.

\section{SIMULATIONS UNDER NON-UNIFORM IRRADIANCE}

To show the capabilities of this improved model, simulations under non-uniform irradiance have been done. For the sake of clarity, Gaussian light profiles have been used. Figure 6 represents the three Gaussian profiles simulated, with peaks of $3650 \mathrm{X}, 3700 \mathrm{X}$ and $10000 \mathrm{X}$ over the average of $1000 \mathrm{X}$ in all the cases.

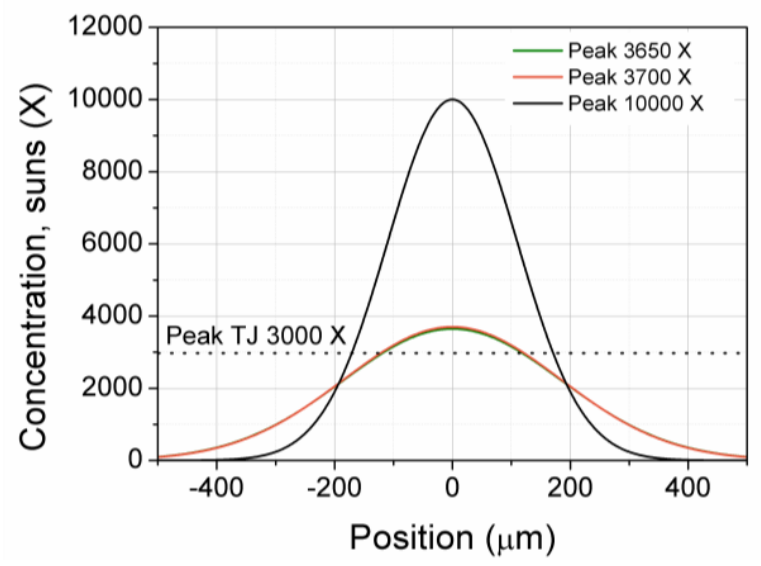

FIGURE 6. Gaussian light profiles simulated, with different peaks over the average of $1000 \mathrm{X}$ in all the cases.

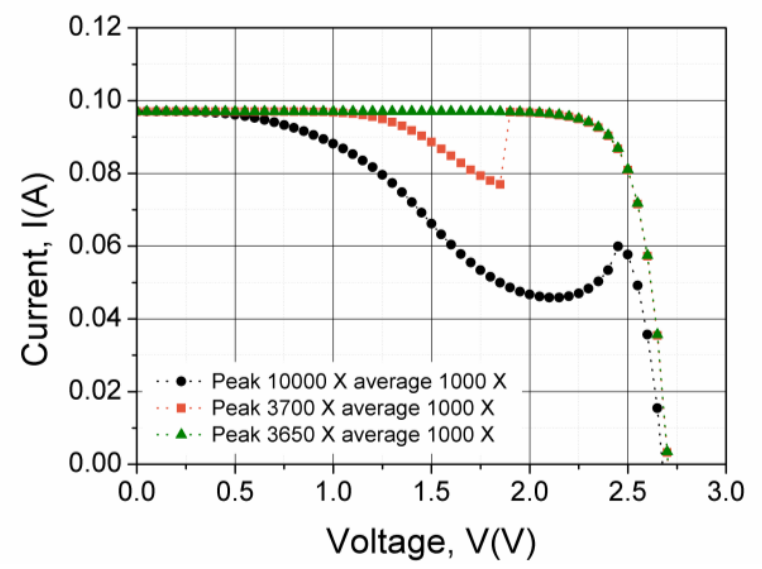

FIGURE 7. I-V curves of the dual-junction under different Gaussian light profiles. 
In figure 7 the I-V curve generated by these light profiles is shown. It has to be noted that the short circuit currents are smaller than $0.135 \mathrm{~A}$, since the Gaussian profiles are circular and consequently leave some active area of the square solar cell in the dark. In figure 7 it is observed that dip-related effects are not observed until a peak of up to $3650 \mathrm{X}$. As expected, Gaussian profiles allow peaks higher than $3090 \mathrm{X}$ since the area surpassing the peak of the tunnel junction is considerably smaller.

\section{SIMULATIONS UNDER CHROMATIC ABERRATION CONDITIONS}

A common problem in solar cells concentrators is the chromatic aberration. In this study we compare the case of a Gaussian light profile with peak $3650 \mathrm{X}$ with and without chromatic aberration. The way of simulating chromatic aberration consists of a top cell impinged by a Gaussian with a peak of $4000 \mathrm{X}$ together with a bottom cell impinged by a Gaussian with peak $3650 \mathrm{X}$. Both subcells with an average of $1000 \mathrm{X}$. Although at high concentrations, the bottom cell is the limiting subcell (with a peak of $3650 \mathrm{X}$ ), figure 8 reveals that this chromatic aberration originates a dip in the I-V curve, since the current spreading is unable to maintain the whole tunnel junction working in the tunnelling region.

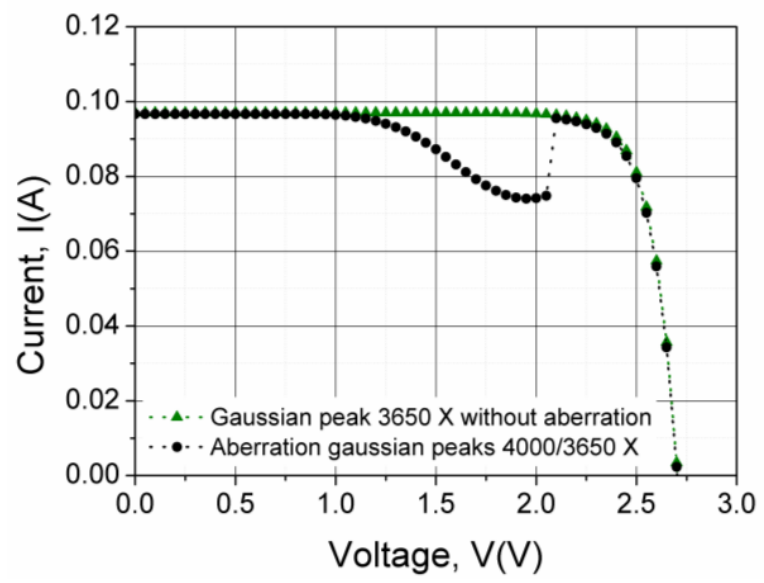

FIGURE 8. I-V curves of the dual-junction with and without chromatic aberration.

\section{CONCLUSION}

This paper has reported a distributed simulation method for multi-junction concentrator solar cells in which the tunnel junction is fully described. To asses the potential of the method simulations under uniform, non uniform and chromatic aberration conditions were preformed. The existence of a non negligible lateral current in the anode of the tunnel junction was shown. This current is responsible for not originating a dip in the I-V curve under certain circumstances where the solar cell short circuit current is higher than the tunnel junction peak current.

\section{ACKNOWLEDGMENTS}

This paper was supported by the Spanish Ministerio de Educación y Ciencia under the CONSOLIDER-INGENIO 2010 program by means of the GENESIS FV project (CSD2006-004) and the research projects with references TEC2009-11143, TEC2008-01226 and PSE-440000-2009-8. The Comunidad de Madrid has also contributed under the NUMANCIA program (S-0550/ENE/0310).

\section{REFERENCES}

1. I. García, I. Rey-Stolle, B. Galiana, C. Algora, Appl. Phys Lett 94 (5) (2009) 053509.

2. J. F. Geisz, D. J. Friedman, J.S. ward, W. J. Duda, Appl Phys Lett 93 (12) (2008) 123505

3. W. Guter, J. Schone, S. P. Philipps, G. Steiner, M. Siefer, A. Wekkeli, E. Welser, , E. Oliva, A. W. Bett, F. Dimroth, Appl Phys Lett 94 (22) (2009) 223504.

4. R. R. King, D. C. Law, K. M. Edmondson, G. S. Fetzer, C. M.and Kinsey, H. Yoon, R. A. Sherif, N. H. Karam, Appl Phys Lett 90 (18) (2007) 183516.

5. R. Winston, J. C. Miñano, P. Benítez "Nonimaging Optics" Elsevier, Oxford, 2005, pp. 318-327, 363-366

6. W. Guter, A.W. Bett IEEE Trans Electron Devices 53 (9) (2006) 2216-2222.

7. M. Victoria, C. Domínguez, I. Antón and G. Sala Optics Express, Vol. 17, No. 8, (2009)

8. I. Rey-Stolle, C. Algora, I. Garcia, M. Baudrit, P. Espinet, B. Galiana, E. Barrigon, 35th IEEE Photovoltaic Specialist Conference, IEEE, Philadelphia USA, 2009.

9. K. Nishioka, T. Takamoto, W. Nakajima, T. Agui, M. Kaneiwa, Y. Uraoka, T. Fuyuki, Proceedings of 3rd World Conference on Photovoltaic Energy Conversion, Vols a-C (2003) 869-872.

10. B. Galiana, C. Algora, I. Rey-Stolle, Progress in Photovoltaics 16 (4) (2008)331-338.

11. S. M. Sze, Physics of semiconductor devices, 2nd Edition, Wiley, New York, 1981.

12. Universidad Politécnica de Madrid, Método implementado en computador para la simulación numérica de un dispositivo semiconductor que comprende uniones túnel, patent application EP10382076.7

13. A. Braun, B. Hirsch, E.A. Katz, J. M. Gordon, W. Guter, A.W. Bett,Sol Energy Mater Sol Cells 93 (9) (2009) 1692-1695 